\title{
Complicated Case
}

\section{Magnetic Seizure Therapy Improves Mood in Refractory Major Depression}

\author{
Markus Kosel', Caroline Frick', Sarah H Lisanby', Hans-Ulrich Fisch' and Thomas E Schlaepfer*,1,2 \\ 'Psychiatric Neuroimaging Group, Department of Psychiatry, University Hospital Bern, Switzerland; 'Brain Stimulation Group, Department of \\ Psychiatry, University Hospital Bonn, Germany
}

\begin{abstract}
This report describes the successful treatment of a patient suffering from an episode of drug-resistant major depression using magnetic seizure therapy (MST). The patient suffered from recurrent major depression since adolescence. MST is a novel brain stimulation method using transcranial magnetic stimulation at convulsive parameters in order to induce therapeutic seizures under general anesthesia in the same setting used for electroconvulsive therapy (ECT). The first use of therapeutic magnetic seizure induction in a psychiatric patient took place at the University Hospital in Bern, Switzerland, in May 2000. Results of a recent randomized, within-subject, double-masked trial comparing ECT and MST in 10 patients indicate that MST appears to have less subjective and objective side effects, is associated with faster recovery of orientation, and is superior to ECT on measures of attention, retrograde amnesia, and category fluency. ECT has an unparalleled and well-documented efficacy in severe depression but is associated with cognitive side effects. MST is currently under study in several centers with respect to its antidepressant efficacy. We report here on the treatment of a patient with refractory major depression (DSM IV-R), who underwent a series of 12 sessions of MST in an inpatient setting. Baseline Hamilton Depression Rating Scale (HRSD-2 I) of 33 and Beck Depression Inventory (BDI) of 40 decreased to 6 and II respectively, I week after completion of the MST trial. Measures of cognitive functions support the hypothesis that MST is associated with a less severe profile of cognitive side effects. $\left[{ }^{99 \mathrm{~m}} \mathrm{Tc}\right]-\mathrm{HMPAO}$ SPECT studies (baseline and 4 days after the completion of the MST trial) point to a raise of blood flow at baseline in the left fronto-parietal region and the brainstem. Our preliminary data support the prospect of antidepressant efficacy of MST and point to a benign cognitive side-effect profile in a patient suffering from severe treatment-resistant major depression.

Neuropsychopharmacology (2003) 28, 2045-2048, advance online publication, 27 August 2003; doi: I 0.1038/sj.npp. I 300293
\end{abstract}

\section{CASE PRESENTATION}

The 66-year-old female patient was referred to our electroconvulsive therapy (ECT) treatment unit by her general practitioner. She was suffering since age 17 from recurrent major depressive episodes with comorbid anorexia nervosa and obsessive-compulsive disorder. She was only free of any depressive symptoms during 1 year 20 years prior to the reported treatment course. The patient had to retire from work and got a full pension because of her incapacity to work as a secretary due to the major depression 6 years later. The current episode of depression started 5 years ago, after a short period of partial remission lasting several months only. During this episode, she received psychotherapy and the following psychotropic medication: citalopram $10 \mathrm{mg} /$ day and venlafaxine $18.75 \mathrm{mg} /$ day, which had to be stopped because of intolerable side effects after 1 week of treatment. A 5-month trial

\footnotetext{
*Correspondence: Dr TE Schlaepfer, Department of Psychiatry, University Hospital, Sigmund-Freud-Strasse 25, 53105 Bonn, Germany, Tel: + 49228 287-57|5, Fax: + 49228 287-5025,

E-mail: schlaepf@jhmi.edu

Received 16 June 2003; accepted 10 July 2003

Online publication: 18 July 2003 at http://www.acnp.org/citations/ Npp07190303264/default.pdf
}

with a standardized preparation of St Johns Wort, up to $900 \mathrm{mg} /$ day, was stopped because of ineffectiveness. Then the patient was treated with nefazodone at doses up to $900 \mathrm{mg} /$ day during 50 months prior and during the magnetic seizure therapy (MST) trial, with the following additional: amisulpiride up to $100 \mathrm{mg} /$ day during 5 months, olanzapine $1.25 \mathrm{mg} /$ day during 1 week which had to be stopped because of side effects, $0.5-1 \mathrm{mg} /$ day risperidone during 26 months prior and during the MST trial, carbamazepine $100 \mathrm{mg} /$ day during 5 months, and sporadically lower doses of lorazepam. The psychotropic medication was not changed during the 2 months before and during the MST-treatment course.

In general, the treatment of this patient was difficult because of the complex and long-lasting comorbid psychiatric disorders. The patient interrupted several times drug trials with different tricyclic drugs because of unbearable side effects (nausea, and especially because of fear of weight gain). According to her general practitioner, she was reluctant to change medications to drugs unknown to her.

\section{METHODS}

After the baseline assessment ( 35 days before the start of the MST trial), the patient was enrolled in the MST trial under a 
protocol approved by the Institutional Review Board of the State of Bern, Switzerland, and according to the Helsinki Declaration (WMA, 2000). At that moment, a Structured Clinical Interview according to DSM IV (SCID-I, German version, Wittchen et al, 1997) led to the following psychiatric diagnosis: recurrent major depressive episode, with melancholic features, severe, without psychotic symptoms (DSM IV 296.33), anorexia nervosa, binge-eating/ purging type, partial remission (DSM IV 307.1), obsessivecompulsive disorder (DSM IV 300.3), with mainly washing and checking rituals. Somatic diagnoses were fenestration of vertebrae L4-S1 and decompression of S1, left side, 17 years earlier, Morbus Scheuermann during adolescence. The severity of the symptoms of depression was assessed with the Beck Depression Inventory (BDI, self-rating, German adaptation of Beck, 1987) and the Hamilton Rating Scale of Depression (HRSD-21, German adaptation of Hamilton, 1967) at baseline (35 days before the start of the MST trial, after MST session 4,10 and 12, and 1 week after the trial). The following cognitive tests were performed at baseline and at follow-up (1 week after the MST trial): verbal learning and remembering (Münchner Verbaler Gedächtnistest, Ilmberger, 1988) (a German research adaptation of the California Verbal Learning Test, Delis et al, 1987); nonverbal learning and remembering (Rey Visual Design Learning Test, Rey, 1964). To measure the recovery of orientation after every seizure induction, we assessed the time needed to answer correctly four out of five questions (name, place, day of the week, age, date of birth) after recovery of spontaneous respiration according to Sobin et al (1995). To assess immediate cognitive performance before and immediately after ( $5 \mathrm{~min}$ after regaining of orientation) MST trials 1, 4, 8, 10, and 12, we used tests similar (German words) to the treatment effect battery (TEB) as proposed by Sackeim et al (1986, 1993). Our battery contained an assessment of verbal and nonverbal learning and recall task as measures for retrograde amnesia. Treatments were delivered with a custom-modified magnetic stimulator, among other modifications using 16 instead of four booster modules (Magstim Super Rapid; Magstim Company, Ltd, Whitland, Wales). The peak magnetic field induced is $2 \mathrm{~T}$ at the coil surface, according to the operating manual. The pulse has a dampened cosine waveform with a pulse width twice as wide as the unmodified rTMS device by the same manufacturer. Stimulation parameters were $50 \mathrm{~Hz}, 8 \mathrm{~s}$, at maximal output. Treatments were administered using a standard butterfly coil (outer diameter of the cones $13 \mathrm{~cm}$ ). During stimulation the center of the coil was placed at the vertex.

Anesthesia was performed with $0.5-1 \mathrm{mg}$ alfentanil and $0.33-0.48 \mathrm{mg} / \mathrm{kg}$ etomidate according to the ECT treatment protocol used at our facility. Muscle relaxation was induced with $0.93-1.4 \mathrm{mg} / \mathrm{kg}$ of succinylcholine. The patient was oxygenated with $100 \% \mathrm{O}_{2}$ at least $5 \mathrm{~min}$ before induction of anesthesia and during the whole anesthesia. Left frontomastoid electroencephalogram (EEG) and the movement of the right leg (which was cuffed prior to the administration of the muscle relaxant agent) were recorded with an MECTA 5000 ECT stimulator during and after magnetic seizure induction.

Two $\left[{ }^{99 \mathrm{~m}} \mathrm{Tc}\right.$ ]-HMPAO SPECT studies were performed, one baseline recording 5 days before the start of the MST trial and another 4 days after the last MST session. Data were recorded using a Phillips Irix triple-head camera with a VXHR collimator. Prior to injection of $550 \mathrm{MBq}$ [99m $\mathrm{Tc}$ ]HMPAO $\left(\right.$ Ceretec $\left.^{\mathbb{R}}\right)$, the patient rested during 30 min in a dark, quiet room in order to reduce the possibility of nonspecific activation. Data acquisition followed $60 \mathrm{~min}$ after the injection of the tracer.

\section{RESULTS}

At baseline, depression was rated on the BDI with 40 and on the HRSD-21 with 33 . These scores decreased steadily during the MST trial to reach values of 11 and 6, indicating remission (Figure 1).

The 12 MST sessions were tolerated well, and no adverse events especially headache, muscle pain, and subjective memory impairment were reported. Duration of tonicclonic movement varied between 14 and $23 \mathrm{~s}$, with the exception of the second treatment session, where no clonictonic seizure could be induced. A recording of a typical seizure is presented in Figure 2 (MST session 9). Recovery of orientation after the induction of a seizure measured from the moment of spontaneous respiration occurred at $4.8 \pm 2.9$ min (mean of the 12 MST sessions \pm SD). This time is significantly shorter than the that reported after unilateral low-, moderate- and high-dosage ECT and after bilateral high-dosage ECT where time to orientation was $18.7,17.1,30.7$, and $45.5 \mathrm{~min}$, respectively (Sackeim, 2000). Measures of retrograde amnesia (tests similar to the TEB battery) of verbal and nonverbal learning and recall yielded the following results: before the induction of anesthesia the patient recalled $8.4 \pm 1.6$ words and $6 \pm 1.2$ figures, whereas immediately after the treatment she recalled $7 \pm 3.0$ words (20\% decrease) and $6 \pm 1.5$ figures (mean of assessments of MST sessions 1, 4, 8, 10, and $12 \pm$ SD). No significant decrease was found neither for verbal

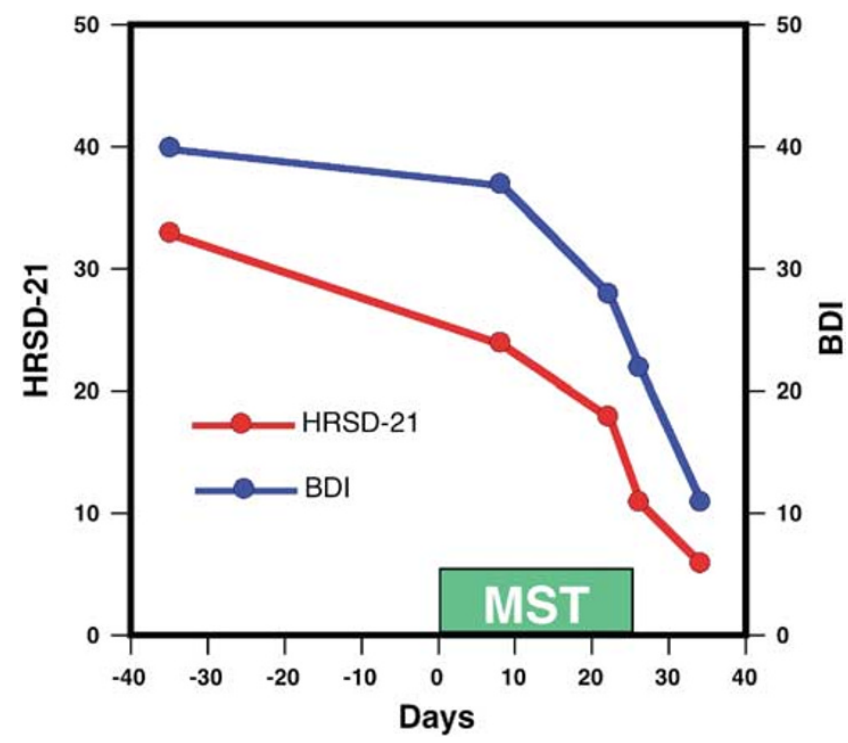

Figure I Rating of the severity of symptoms of depression: Beck Depression Inventory $(\mathrm{BDI})$ and Hamilton Rating Scale of Depression (HRSD-2I) scores are reported at baseline (35 days before the start of the MST trial) and $6 \mathrm{~h}$ after MST sessions 4, 10, and 12, and I week after the end of the MST trial. 


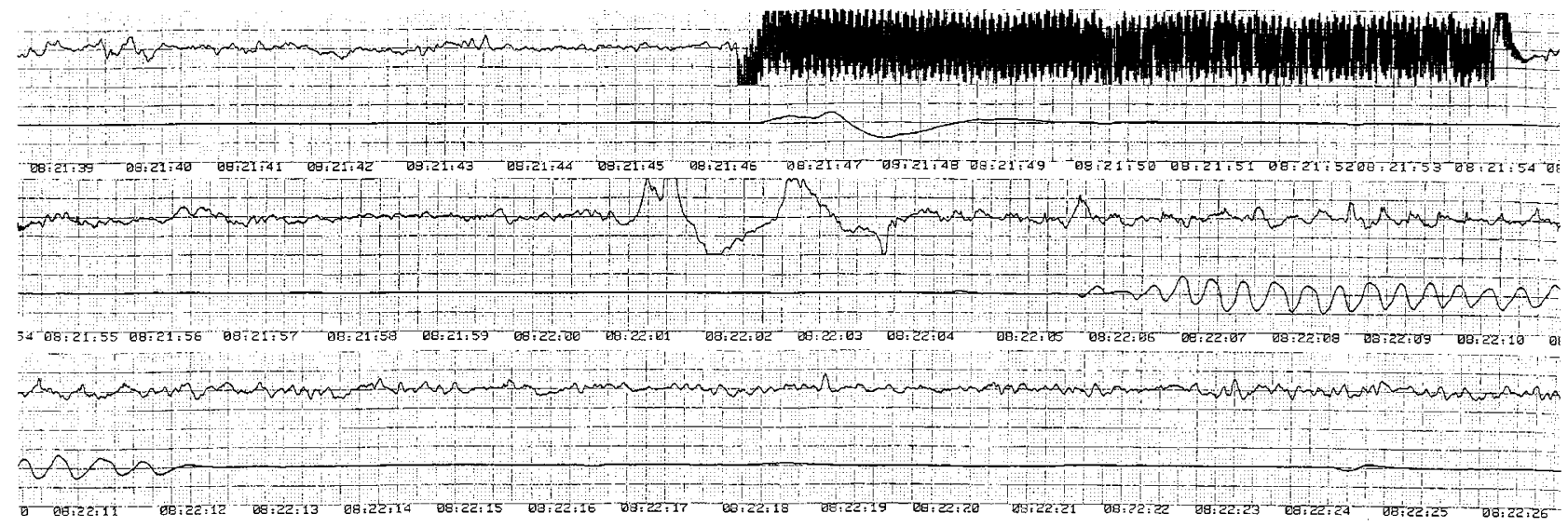

Figure 2 Left fronto-mastoid EEG and movement (right cuffed foot) recordings of MST session 9. These recordings were performed with an MECTA 5000 ECT stimulator during and after magnetic seizure induction. The upper trace represents the EEG record. The high-amplitude parts of $8 \mathrm{~s}$ are MST stimulation artifacts. The lower trace is the optical movement sensor recording.

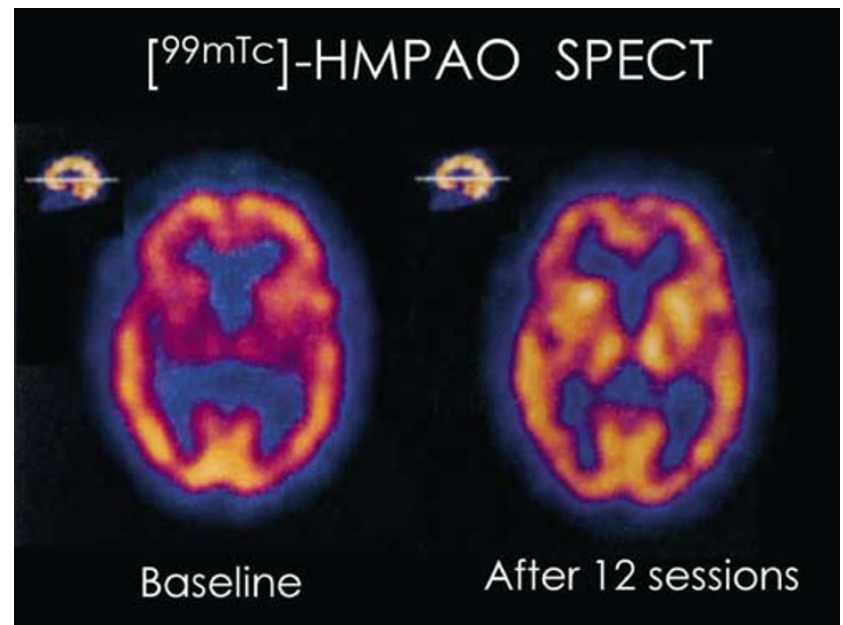

Figure $3 \quad\left[{ }^{99 m} \mathrm{Tc}\right]-\mathrm{HMPAO}$ SPECT recordings (Phillips Irix 3-head camera with a VXHR collimator, $550 \mathrm{MBq}\left[{ }^{99 \mathrm{~m}} \mathrm{Tc}\right]-\mathrm{HMPAO}$ (Ceretec)) 5 days before the start of the MST trial (left) and 4 days after (right). Bright colors indicate higher cerebral blood flow.

nor nonverbal material ( $p=0.28$ and $1, t$-test). Compared to the study by Sackeim (2000), our values were lower. In that study, the decrease reported amounted to 84.3, 90.6, 93.5, and $95.2 \%$ in the verbal task and $29.5,32.0,36.0$, and $41.8 \%$ in the visual task after unilateral low-, moderate-, and highdose ECT and after bilateral high-dose ECT, respectively.

At baseline, verbal learning performance (Münchner Verbaler Gedächtnistest) was 8.6 items \pm 2.5 and nonverbal learning 7.0 items \pm 2.2 , whereas the respective values after the MST trial were 12.4 items \pm 3.2 and $10.2 \pm 2.6$ (means $\pm \mathrm{SD}$ ). These differences were statistically significant ( $t$-test, $p=0.003$ and 0.001 respectively, pre-compared to post-treatment). The SPECT studies demonstrated a higher perfusion of the fronto-parietal cortex and the basal ganglia after the treatment compared to baseline (Figure 3).

\section{DISCUSSION}

We report on the efficacy and the cognitive side effects of a treatment course with 12 sessions of MST. The MST treatment was performed very much like ECT, the only difference was the application of magnetic $v s$ electric stimulation in order to induce seizures. The patient, suffering from a treatment-resistant episode of major depression lasting 5 years, reached after 4 weeks of treatment remission of depressive symptoms as indicated by BDI- and HRSD-21 scores. A total of 11 short tonicclonic seizures could be elicited during the MST trial. The seizure recording of session 9 is shown in Figure 2 . Movement recordings indicate a short tonic-clonic seizure compared to seizures elicited by therapeutic ECT courses. The EEG recordings in general and also those shown in Figure 2 are not suggestive of comparable seizure activity (spikes and waves) to those usually recorded during ECT, very much like the findings of Lisanby et al (2003, 2001). The treatment was tolerated well and no side effects were reported, especially no subjective cognitive impairments. The cognitive measures assessed in this study indicate less severe objective cognitive side effects than those reported after ECT treatment (Lisanby et al, 2003; Sackeim, 2000). Verbal and nonverbal learning tasks improved significantly from baseline compared to 1 week after completion of the MST trial. A SPECT study indicated an increased cerebral blood flow in the frontoparietal region and the basal ganglia, regions in which the cerebral activity is impaired during depression (Drevets, 2000), 4 days after 4 weeks of MST treatment compared to the baseline assessment.

Additional studies are certainly needed to confirm the findings of this case report. If they confirm that MST has significant antidepressant effects while maintaining a benign cognitive side-effect profile, the method could be seen as a major advance in the treatment of refractory major depression possibly complementing or even replacing ECT.

\section{REFERENCES}

APA (2001). ECT Task Force of the American Psychiatric Association: The Practice of Electroconvulsive Therapy. American Psychiatric Press: Washington, DC.

Beck A (1987). Beck Depression Inventory: Manual. Psychological Corporation: San Antonio, TX. 
Delis DC, Kramer JH, Kaplan E, Ober BA (1987). California Verbal Learning Test: Adult Version Manual. The Psychological Corporation: San Antonio, TX.

Drevets WC (2000). Functional anatomical abnormalities in limbic and prefrontal cortical structures in major depression. Prog Brain Res 126: 413-431.

Hamilton M (1967). Developement of a rating scale for primary depressive illness. Br J Soc Clin Psychol 6: 278-296.

Ilmberger I (1988). Münchner Verbaler Gedächtnistest (unpublished German adaptation of the California Verbal Learning Test).

Lisanby HS, Luber B, Schlaepfer TE, Sackeim HA (2003). Safety and feasibility of magnetic seizure therapy (MST) in major depression: randomized within-subject comparison with electroconvulsive therapy. Neuropsychopharmacology 16 July 2003; e-pub. doi: 10.1038/sj.npp.1300229.

Lisanby SH, Schlaepfer TE, Fisch HU, Sackeim HA (2001). Magnetic seizure therapy of major depression. Arch Gen Psychiatry 58: 303-305.
Rey A (1964). L'Examen Clinique en Psychologie. Presses Universitaire de France: Paris.

Sackeim HA (2000). Memory and ECT: from polarization to reconciliation. J ECT 16: 87-96.

Sackeim HA, Decina P, Prohovnik I, Portnoy S, Kanzler M, Malitz S (1986). Dosage, seizure threshold, and the antidepressant efficacy of electroconvulsive therapy. Ann NY Acad Sci 462: 398-410.

Sackeim HA, Prudic J, Devanand DP, Kiersky JE, Fitzsimons L, Moody BJ et al (1993). Effects of stimulus intensity and electrode placement on the efficacy and cognitive effects of electroconvulsive therapy. N Engl J Med 328: 839-846.

Sobin C, Sackeim HA, Prudic J, Devenand DP, Moody BJ, McElhiney MC (1995). Predictors of retrograde amnesia following ECT. Am J Psychiatry 152: 995-1001.

Wittchen HU, Wunderlich U, Gruschwitz S, Zaudig M (1997). SKID I: Strukturiertes klinisches Interview für DSM-IV. Hogrefe: Göttingen.

WMA (2000). World Medical Association: Declaration of Helsinki: Ethical Principles for Medical Research Involving Human Subjects. EWMA: Edingburgh. 